\title{
How effective is community mobilisation in HIV prevention among highly diverse sex workers in urban settings? The Aastha intervention experience in Mumbai and Thane districts, India
}

\author{
Sanjeev Singh Gaikwad, ${ }^{1}$ Amrita Bhende, ${ }^{1}$ Gaurav Nidhi, ${ }^{1}$ Niranjan Saggurti, ${ }^{2}$ \\ Virupax Ranebennur ${ }^{1}$
}

${ }^{1} \mathrm{FHI}$ 360, Mumbai, India ${ }^{2}$ Population Council, New Delhi, India

Correspondence to Dr Sanjeev Singh Gaikwad, FHI 360 Maharashtra, 501-505 Balarama Building, Bandra-Kurla Complex, Bandra East, Mumbai 400 051, India;

sgaikwad@fhi360.org

Accepted 21 May 2012

Published Online First

3 July 2012

\section{UNLOCKID}

This paper is freely available online under the BMJ Journals unlocked scheme, see http:// jech.bmi.com/site/about/ unlocked.xhtml

\section{ABSTRACT \\ Background This paper examines the association} between degree of confidence in collective efficacy and self-efficacy for condom use and empowerment among heterogeneous female sex workers (FSWs) in two metropolitan Indian cities with high HIV prevalence. Methods The study utilises data from the Behavioural Tracking Survey, a cross-sectional behavioural study with 2106 FSWs recruited from 411 intervention sites in Mumbai and Thane. The key independent measures used determine the degree of confidence in collective efficacy (belief in the power to achieve goals and address problems together) and outcome measures included: self-efficacy for condom use with occasional clients and condom use with regular partners, self-confidence in handling a crisis situation and public speaking ability. Univariate and multivariate statistical methods were used to examine the study objectives.

Results Of the analytical sample of 2106 FSWs, 532 (25.3\%) reported high degree of collective efficacy for achieving certain goals and 1534 (72.8\%) reported collective efficacy for addressing specific problems. FSWs reporting a higher collective efficacy as compared with those reporting lower collective efficacy were as follows: more likely to negotiate condom use with occasional clients (60.3\% vs 19.7\%; adjusted OR (AOR) $=6.3,95 \% \mathrm{Cl} 4.8$ to 8.4 ) as well as regular partners (62.8\% vs $20.2 \%$; $\mathrm{AOR}=6.4,95 \% \mathrm{Cl} 4.9$ to 8.4$)$ : confident in facing troublesome stakeholders $173.5 \%$ vs $38.8 \%$; $\mathrm{AOR}=4.3,95 \% \mathrm{Cl} 3.3$ to 5.6$)$, confident in supporting fellow FSWs in a crisis $(76.1 \%$ vs $49.6 \%$; AOR $=2.9,95 \% \mathrm{Cl} 2.2$ to 3.7), received help from other FSWs when a client or partner was violent $173.9 \%$ vs $46.3 \%$; $\mathrm{AOR}=3.5,95 \% \mathrm{Cl} 2.7$ to 4.5 ) and had stood up to the police or madams/brokers to help fellow FSWs in the past 1 year (5.8\% vs 3.3\%; $\mathrm{AOR}=2.7,95 \% \mathrm{Cl} 1.5$ to 4.9).

Conclusion The results suggest that the strategy of collectivisation in HIV prevention programme has much broader benefits than merely the promotion of safer sex practices. Future HIV prevention interventions in India and elsewhere may include collectivisation as the core strategy within HIV prevention programmes.

\section{INTRODUCTION}

Recent studies reflect a shift in the focus of HIV/ AIDS prevention efforts from models aimed at individual risk behaviour change to models aimed at community mobilisation and empowerment as a result of growing awareness of the complex social, cultural, political and economic forces shaping the HIV epidemic. ${ }^{1-3}$ While literature has shown that interventions that build community collectives can empower vulnerable populations to negotiate safe sex practices, ${ }^{4}$ little is known about the effect of collective efficacy on self-efficacy and the confidence of female sex workers (FSWs) in times of crisis and related condom usage. ${ }^{156}$

The Sonagachi programme in Kolkata, India, for example, organised FSWs into a community-based organisation (CBO) and involved them in implementing HIV prevention activities. ${ }^{4}$ In addition to raising FSWs' social and economic status, the programme empowered them over time, resulting in improved negotiation for condom use and more equitable relationships with clients, reduced police brutality and reduced prevalence of HIV. ${ }^{4-11}$

Over the time, the Sonagachi sex workers established a CBO, the Durbar Mahila Samanwaya Committee (DMSC) 'which organises weekly and monthly problem-solving meetings, and promotes sex workers' social and political awareness through critical discussions of the stigma attached to sex work, and the value of collective action'. ${ }^{12}$ (p.499) DMSC showed that involving FSWs directly in organisational activities and empowerment strategies reduces their vulnerability to HIV/STIs as compared with programmes that provide only clinical and prevention services. ${ }^{6}$ 8-11 1314 The success of the Sonagachi programme can be attributed to the active participation of sex workers in decision-making and programme implementation through the DMSC. ${ }^{6} 10^{11}$ With exception to Sonagachi programme among brothel-based sex workers in Kolkata city in India, studies have identified the disperse, hidden, highly controlled and mobile nature of local sex trades as barriers to empowerment of the community. ${ }^{15}$

This paper focuses on the Aastha project, an upscaled HIV prevention intervention with FSWs from varied cultures and religions, who are highly mobile, geographically dispersed and solicit clients from multiple locations including brothels, bars, streets, beaches, homes and through phone networks. The Aastha project was implemented by Family Health International (FHI) 360 in two districts of Maharashtra (Mumbai and Thane), which also have high HIV prevalence and high numbers of FSWs. ${ }^{16}$ This paper describes the process adopted by Aastha, as part of Avahan 
initiative $^{17}$ to promote FSW' confidence in collective efficacy and build FSWs' self-esteem, self-efficacy for condom use and negotiation skills to help others in times of crisis. We hypothesise that FSWs expressing higher confidence in collective efficacy are more likely than others to demonstrate self-efficacy for condom use and confidence in handling issues that concern sex workers.

\section{FSWs' context in Mumbai and Thane districts}

Mumbai and Thane, the two most populated districts of India, located in Maharashtra state, are also known to be hubs for business, arts and the media. The 2011 Census for the Mumbai Metropolitan Region recorded a population of 12.5 million and Thane recorded 11.1 million. Being economically active, both districts provide housing and livelihoods to over 35000 FSWs per district, which is 10 times more than any other Indian district. ${ }^{18}$

Of the 20606 FSWs in the Aastha programme implementation sites, 3035 FSWs solicit clients in brothels, 7946 in bars, 5061 in streets and 4564 in homes. Many brothel-based FSWs operate out of small rooms used by 3-4 FSWs as their living and workplace. Almost all brothel keepers get a $50 \%$ share of the FSWs' earnings. Almost two-thirds of brothel-based FSWs are from less developed districts of Karnataka, Andhra Pradesh, Bengal and Nepal. Brothel keepers tend to exercise control over FSWs' lives, including condom usage and the provision of sexual services to clients. Pimps thrive where FSWs do not have direct access to clients for negotiations, such as in the bungalow system of brothels in Kamathipura, and are paid $25 \%-30 \%$ of FSW's fees. Street-based FSWs are mainly in the lowest socioeconomic strata and are at the mercy of pimps, local goons and the police, who are the main perpetrators of violence. FSWs working in bars present themselves as waitresses or singers and give a major percentage of their earnings to the bar manager. In contrast, home-based FSWs (who solicit clients through a phone network of pimps) are a hidden and hard-to-reach population.

As many FSWs are migrants from other states, mobility is high especially periodic visits to their native place. ${ }^{19} \mathrm{HIV}$ prevalence among FSWs in Mumbai and Thane exceeded 30\% in $2006 .{ }^{20}$ Although consistent condom use with occasional clients was high (Mumbai 98\% and Thane 95\%), it was low with regular partners (Mumbai 35\% and Thane 34\%). ${ }^{21}$ These factors, combined with a high inflow of new FSWs entering these districts, necessitated a unique community mobilisation process.

\section{Programme description: the Aastha project}

Given the multicultural and large concentration of FSWs with heightened vulnerabilities, the Aastha HIV/STI prevention programme was initiated in 2004 with technical guidance from FHI 360. The objective of the Aastha project was to promote individual FSW empowerment to access and manage HIV prevention services, a strategy that went beyond the traditional approaches of community collectivisation due to the fact that the FSWs are multicultural, distributed across a wide geography and solicit clients in different types of places. Details of community mobilisation intervention strategies by each typology of sex work are presented in table 1. The Aastha project initiated the programme in select areas covering around 30000 FSWs of a total estimated 60000 FSWs in Mumbai and Thane districts. The remaining FSWs were covered by the government's National AIDS Control Programme under the auspices of the State AIDS Control Society.

In order to empower FSWs, the Aastha project capacitated the project team consisting of peer educators and sex workers who were given an honorarium to initiate self-help groups, also called Aastha Gats (AGs), at the local level (as shown in figure 1). About 2-3 self-help groups (AGs) of FSWs were formed within the catchment area of 50 FSWs. Each AG had non-paid (volunteer) members. The voluntary AG members and leaders of respective AGs went on to form $\mathrm{CBOs}$ at the subdistrict level and the Aastha Parivaar, a federation of these CBOs at the project level. AG members placed emphasis on promoting unity as needed among FSWs within defined geographical areas. The AG members formed a core group to work together on issues that concerned the community. The $\mathrm{CBOs}$ and the Aastha Parivaar focused on building the capacity of AG members on collectivisation for decision making, problem solving, leadership and programme and crisis management as individuals and as a group, as well as organising large-scale events in the districts.

\section{METHODS}

\section{Study design}

This paper utilises data from the Behavioural Tracking Survey, a cross-sectional behavioural survey conducted among FSWs to monitor the key components of the HIV prevention programme which included safe sex behaviour, sexually transmitted infection, treatment-seeking behaviours and community mobilisation. Survey participants were recruited from 411 sites in the two project implementation districts, Mumbai and Thane.

Survey respondents included women who were $\geq 18$ years who had sex in exchange of cash/kind in the 1 month prior to the survey. The Aastha project actively registers each FSW in all sites and provides weekly services. FSWs migrating do not remain in the demonstration data and new FSWs are added within a week of entering any site. Thus, registration includes FSWs physically present and receiving services. FSWs for the survey were chosen from the programme registration data. Prior to sampling, the registration data were verified in the field to obtain the active status of FSWs in the registered list. The list of active members in each cluster (programme implementation area) formed the sampling frame, and FSWs were selected using a systematic sampling procedure.

A sample size of 400 FSWs was estimated allowing for the detection of an absolute difference of $15 \%$ or more from the assumed value of $50 \%$ for consistent condom use with all clients, with $95 \%$ confidence and $80 \%$ power. A design effect of 1.7 was assumed for cluster sampling, and the sample weights were calculated at the site as well as district levels. Since Mumbai and Thane districts have a large number of FSWs according to the following typologies of sex work: brothel based, street/home based and bar based, it was decided to select 400 FSWs from these three major types. From the two districts, a total of 2432 FSWs were interviewed. Of these, data were missing for 326 FSWs on the key variables considered in this paper either due to incomplete interviews or their unwillingness to share information. This resulted in an analytical sample of 2106 FSWs.

Interviews were conducted by trained researchers with verbal and written skills in Hindi, as FSWs belonging to other places and practicing sex work in the study districts understand and speak basic Hindi. In situations where FSWs spoke very little Hindi, researchers who could speak additional languages such as Telugu, Kannada and Marathi were selected to conduct the interview. All the researchers had a bachelor's degree in either sociology or statistics. The survey instrument commonly developed by Avahan for its sites in India ${ }^{1} 1722{ }^{23}$ was modified locally to add a few questions that are relevant to the local 
Table 1 FSW characteristics and community collectivisation strategy by typology of sex work under the Aastha project in Mumbai and Thane, India

\begin{tabular}{|c|c|c|}
\hline Typology & Characteristics of FSWs & Community collectivisation strategy \\
\hline $\begin{array}{l}\text { Brothel- } \\
\text { based } \\
\text { FSWs }\end{array}$ & $\begin{array}{l}\text { Total coverage }=3035 \mathrm{FSWs} \text {; } \\
\text { geographic coverage }=7 \mathrm{~km}^{2} \\
\text { (across } 3 \text { major and other minor } \\
\text { sites); mainly from the states of } \\
\text { Karnataka and West Bengal } \\
\text { as well as Nepal and Bangladesh. } \\
\text { Controlled by brothel managers } \\
\text { and pimps. }\end{array}$ & $\begin{array}{l}\text { Initial collectivisation focused on } \\
\text { increasing legal literacy so as to } \\
\text { reduce police abuse and wrongful } \\
\text { arrest. Another key concern was } \\
\text { reduction of violence by clients, } \\
\text { either due to insistence on sex } \\
\text { without condoms or free sex or } \\
\text { money (regular partners only). }\end{array}$ \\
\hline
\end{tabular}

Bar- $\quad$ Total coverage $=7946$; geographic based coverage of $70 \mathrm{~km}^{2}$; majorly from FSWs the states of West Bengal and Rajasthan and Bangladesh; controlled by bar managers.

Home-

based

FSWs

Total coverage $=4564$ FSWs; geographic coverage $=70 \mathrm{~km}^{2}$; mainly from the states of Bihar, Uttar Pradesh and Maharashtra, controlled by pimps.
FSWs are forced by bar managers to drink alcohol with their clients in order to increase the bills. As a result most FSWs become intoxicated regularly, increasing their vulnerability to unprotected sex. The collectivisation strategy used here was to bring the FSWs together to sensitise the bar managers to their increased risk. Different strategies were created in collaboration with the manager and waiters to reduce the alcohol intake of the FSWs, for example, drinks without alcohol or with minimal alcohol served to the FSWs.

FSWs' transactions are fixed on the phone and therefore sometimes the FSWs do not know their new clients. When the FSW arrives at the designated location, multiple people are present who take advantage of her situation and on occasion do not pay. The collectivisation strategy used initially was to build support groups to prevent or resist violence of this nature.

Challenges

FSWs are largely controlled by brothel managers and pimps.

These stakeholders also keep FSWs separated so as to have greater control over them. As a result, it is difficult to have regular access to FSWs. FSWs have a high transaction frequency and work during odd hours, leaving limited time to access and collectivise them.

Bar managers exercise strict control over access to the FSWs; bar girls work in shifts and rarely meet each other, therefore finding a convenient time for collectivisation is challenging.

FSWs work through the pimp network, largely through the use of mobile phones. As they are a hidden population, they try to protect their identity and so rarely are willing to identify themselves as FSWs or willing to associate with other FSWs for fear of being exposed.

FSWs consider each other to be rivals; they rarely communicate, leave alone come together to support one another. Activities undertaken by Aastha

Sensitisation meetings with brothel keepers and pimps are conducted on the benefits of improved health. Legal literacy sessions and exposure visits to service providers and the local police station are conducted to strengthen community-level linkages and reduce discrimination.

Networking meetings are regularly conducted between self-help group leaders, Bar Association members, bar managers and bar owners to sensitise them to FSWs' issues and needs.

The peer educator plays a crucial role as she provides project services and conducts group activities and events; thereby starting the process of unity without revealing their profession. This process takes a long time. The crisis response system and especially having a group that instantly provides support in times with violence, with regard to family members as well as clients has been the collectivisation strategy. Regular AG meetings are held re-enforcing the need to use the crisis support system.

Regular monthly meetings of those in close geographical proximity are held focusing on sensitisation programmes with local goons (who take regular protection money) as well as legal literacy.

$\begin{array}{ll}\text { Street- } & \text { Total coverage }=5061 \mathrm{FSWs} \text {; } \\ \text { based } & \text { geographic coverage }=70 \mathrm{~km}^{2} \text {; }\end{array}$ FSWs mainly from the state of Maharashtra, controlled by pimps and local goons. goons who regularly take money for protection. These are among the lowest earning FSWs and regular payments to the goons leave them with very little for themselves. Also additionally, the FSWs are dependent on local lodges and hotels to take their clients for sex. Managers change their tariff, sometimes doubling it, leading to much reduced earnings for the FSWs. The strategy used was to build support groups to regularly sensitise lodge owners to reduce exploitation and prevent violence by goons.

FSW, female sex worker.

context. The survey instrument was then translated from English to Hindi. The translated forms were reviewed by the study investigators who were fluent in both English and Hindi. The interview format was then pretested in selected communities.

\section{Data management}

Field staff checked each questionnaire immediately after the interview to ensure accuracy and completion. Field supervisors reviewed the completed questionnaires on the same day, and these were sent every week to the data management team for data entry. A user written computer programme in CSPro (V.4.0) was used for double data entry by trained data entry officers. Data were regularly processed to ensure consistency and accuracy.

\section{Ethical considerations}

The Behavioural Tracking Survey was reviewed and approved by FHI 360's Protection of Human Subjects Committee based in the USA. A local Community Advisory Board was constituted in Mumbai, which worked closely with the local research agency and FHI 360 to ensure that the study participants' rights were protected. A comprehensive informed consent process was followed; respondents were informed about the study, including the duration of the interview of approximately $45 \mathrm{~min}$, and their queries were addressed before written consent was taken. To protect confidentiality and respect privacy, all questionnaires were entirely anonymous and names and addresses were not recorded. To maintain privacy, interviews were conducted in a private or public location, depending on the preference of the respondent. Participants were not given any monetary 


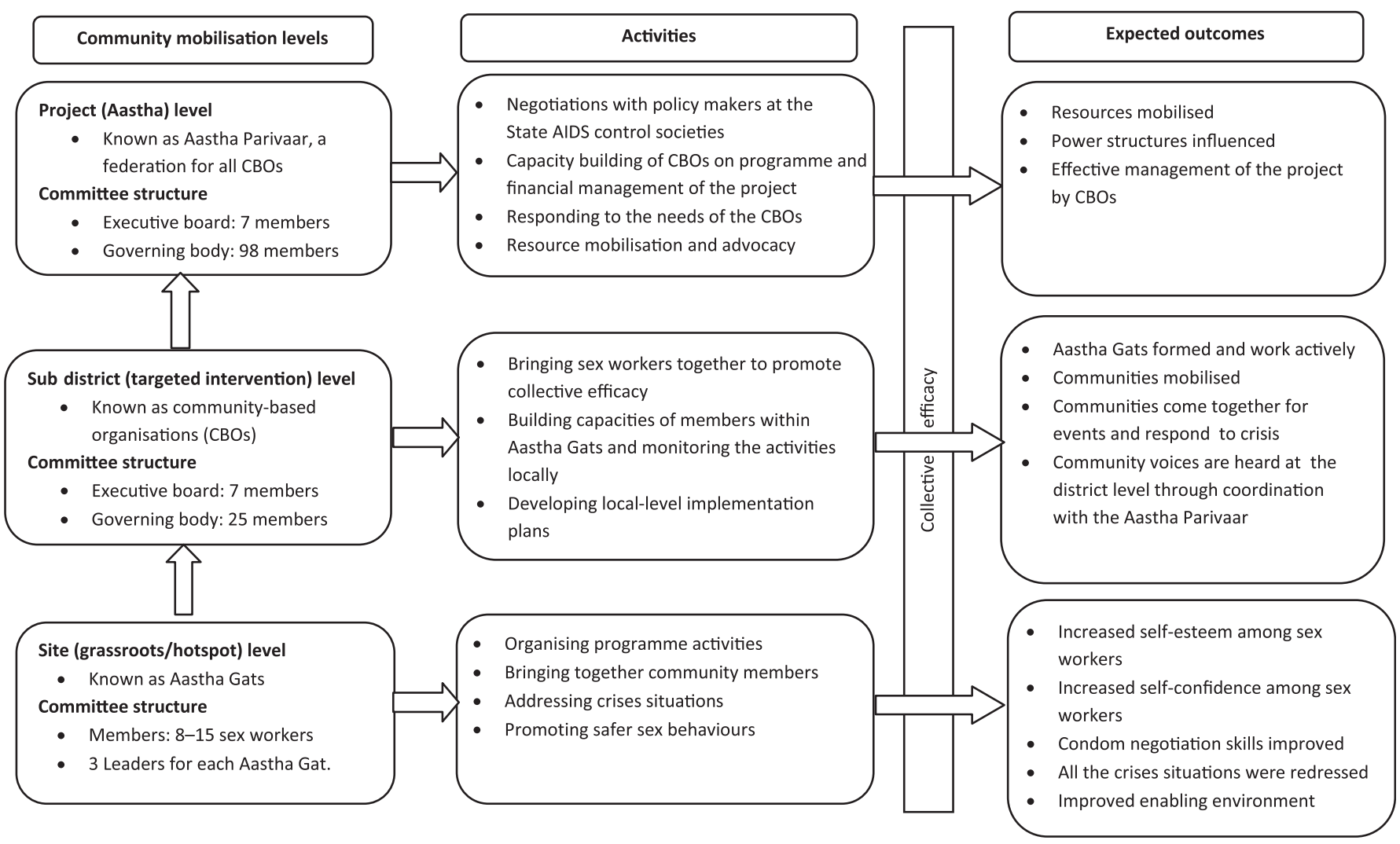

Figure 1 Structure, activities and expected outcomes in the process of community collective efficacy.

compensation for their time in the study but were provided information on HIV programmes in the area.

\section{Measures \\ Socio-demographics}

The variables on socio-demographic characteristics considered in this paper are obtained from single questions in the questionnaire. Responses were coded into two or three categories for analytical purposes. These responses included age (18-29 years, $30+$ years), education (no formal education, formal education), marital status (never married, currently married, formerly married), duration of sex work ( $\leq 0-2$ years, 3-5 years, 6 + years), typology of sex work (brothel based, street/home based, bar based) and district (Mumbai, Thane).

\section{Community collectivisation indicators}

The key independent variables considered in the paper were(1) collective efficacy for achieving certain goals and (2) collective efficacy for addressing specific problems, which are known to influence the behaviours of FSWs.

1. Collective efficacy for achieving certain goals was defined as FSWs confidence in the community to work together to achieve certain shared goals. ${ }^{1}{ }^{24}$ It was measured based on responses to the following questions: How confident are you that FSWs in your community can work together to achieve the following goals: (1) keep each other safe from harm, (2) increase condom use with clients, (3) speak up for your rights and (4) improve your lives. Responses to these questions included: not at all (code: 1), somewhat (code: 2 ), very (code: 3) and completely confident (code: 4). Using these four questions and corresponding responses, an index was constructed, with the scale value ranging from 1 to 4 , which had a reliability (Cronbach's $\alpha$ ) of 0.928 . The index score was further divided into three equal categories of collective efficacy: low (1-1.999), medium (2-2.999) and high (3-4). The higher the score, the higher is the degree of confidence in collective efficacy (referred to as 'collective efficacy to achieve specific goals' in the paper).

2. Another measure of collective efficacy refers to the belief that FSWs can work together for change. It was measured as the willingness of mobilised community members to deal with problems that affected all or some of the FSWs' community. ${ }^{1}$ A question was asked in the survey to measure this: if there was a problem that affected all or some of the FSW community, how many FSWs would work together to deal with the problem? Responses included: no one, some, most and all, and these responses were grouped into two categories: collective efficacy to deal with problems (yes), if the response was either 'most' or 'all'.

The above-mentioned two indicators of collective efficacy were considered separately because they were not strongly correlated to be considered as one domain, as per the results of factor and reliability analyses. For example, the Cronbach's $\alpha$-value for items under both aspects of collective efficacy (all items together) was 0.712 , whereas the exclusion of variable about FSWs addressing specific problems increased the $\alpha$-value to 0.823 suggesting the somewhat weaker relationship of items if they were considered together.

\section{Outcome indicators}

Self-efficacy is defined as the belief that one can accomplish certain goals. The construct of self-efficacy derives from the social cognitive theory. Within this framework, a person is not passive to the environment but instead takes what is there and 
manipulates it to create a self. This active role opposes theories that posit that the environment creates the individual with the individual having no say. ${ }^{25}$ Self-efficacy measure used in this study was multidimensional, including the self-efficacy for condom use, self-efficacy to handle crises and being confident in the FSWs' ability to speak in public and access services.

Each FSW was asked a total of 12 questions to assess her ability to use condoms, negotiate condom use, handle crises, utilise resources, identify herself as a sex worker in a group and confidence in giving her opinion in front of a large group.

Self-efficacy for condom use: three questions assessed selfefficacy for consistent condom use with clients-How confident are you that you can use a condom with each client when (1) he gets angry with you, (2) he offers you more money for sex without a condom, (3) you have been using alcohol or drugs? Further, three questions assessed self-efficacy for consistent condom use with regular partners: How confident are you that you can use a condom with each regular partner when (1) he gets angry with you, (2) you think the risk of disease is low, (3) you have been using alcohol or drugs? Responses to these questions included: not at all (code: 1), somewhat (code: 2), very (code: 3) and completely confident (code: 4). Using these four questions and corresponding responses, an index was constructed, with the scale value ranging from 1 to 4 , which had a reliability (Cronbach's $\alpha$ ) of self-efficacy for condom use with occasional clients (0.692) and regular partners (0.715). The index score was further divided into two categories: low $(1-2.99)$ and high $(3-4)$.

Confidence in handling crises: two questions were asked reflecting FSW's confidence in handling a crisis situation to help herself or other FSWs: Whether or not the FSW was confident in (1) facing alone troublesome stakeholders such as brothel keepers, pimps, police, local goons and (2) supporting fellow sex workers in a crisis? Responses to these questions included: not at all (code: 1), somewhat (code: 2), very (code: 3) and completely confident (code: 4). Sex workers who responded either 'very' or 'completely confident' to the above two questions were considered as confident. Two questions on actual execution of behaviour by respondents were asked: Whether or not the FSW stood up to (1) the police and (2) madams/brokers to help fellow sex workers? Possible responses were no (code: 0 ) and yes (code: 1). Sex workers who said 'yes' to either of the two questions were considered as those who stood up to the police or madams/brokers to help fellow sex workers. A question on whether or not other sex workers helped the respondent when a partner or client was violent was also asked with possible response categories as: no (code: 1), yes (code: 2) and never had a violent partner (code: 3 ).

Other factors that affect FSW' ability to access HIV prevention and care services: the community mobilisation process also included group exposure visits to police stations, meetings with local goons and other key stakeholders such as doctors in public health hospitals. The feeling of togetherness may have led to FSWs' increased confidence and their public speaking ability. These issues were measured in the study using a total of five questions: saying no to a client with whom she is unwilling to have sex, going to a service provider and disclosing her identity as an FSW, travelling alone, giving advice to other FSWs and giving her opinion in front of a large group. Responses to these questions included: not at all (code: 1), somewhat (code: 2), very (code: 3) and completely confident (code: 4). Sex workers who responded as either 'very' or 'completely' confident to the above five questions were considered as confident.
The summary measure of empowerment was constructed by averaging the responses to all the questions used for the above dimensions of self-efficacy and self-confidence. The score varied from 1 to 2 , which had reliability (Cronbach's $\alpha$ ) of 0.78 . The index score was further divided into two equal categories of overall empowerment: low (1-1.4999) and high (1.50-2.0).

\section{Data analysis}

All analyses were conducted to assess the relationship between collective efficacy indicators and the selected outcome variables. Basic descriptive analyses were run on selected socio-demographic characteristics and their association with collective efficacy indicators to describe the sample. Further, both bivariate and multiple variable logistic regression models were used to examine the association between collective efficacy indicators and the outcome variables. All analyses were performed using SPSS software (V.16.0, SPSS Inc.).

\section{RESULTS}

Of the analytical sample of 2106 FSWs, 1502 (71.3\%) reported medium (46\%) and high (25.3\%) collective efficacy for achieving goals, and $1534(72.8 \%)$ reported collective efficacy for addressing specific problems (table 2). These dimensions were not mutually exclusive, and considerable overlap was observed. For example, 475 (22.6\%) FSWs reported both a high degree of collective efficacy for achieving goals and collective efficacy for addressing specific problems, while about $2.7 \%$ reported only a high degree of collective efficacy for achieving goals and $16.8 \%$ reported only collective efficacy for addressing specific problems. FSWs who are currently married than those formerly married $(34.7 \%$ vs $22.3 \%$; $<<0.001)$ and practicing sex work in streets or homes than those in bars $(37.9 \%$ vs $21.2 \%$; $<0.001)$ perceived low degree of collective efficacy for achieving certain goals. Whereas FSWs who have no formal education than those who have formal education (74.6\% vs $68.7 \%$; $<<0.05)$, those formerly married than those currently married $(76.8 \%$ vs $69.1 \%$; $<<0.05)$ and those practicing sex work in brothels than those in streets or homes $(79.0 \%$ vs $68.0 \%$; $<0.001)$ perceived FSWs addressing specific problems.

A greater proportion of formerly married FSWs than unmarried FSWs $(p<0.001)$, brothel- and bar-based sex workers than street/home based $(p<0.001)$ and FSWs from Thane than those in Mumbai $(p<0.001)$ reported medium or high degree of collective efficacy for achieving goals. A higher proportion of FSWs without formal education than those with formal education $(p<0.005)$, formerly married than unmarried $(p<0.001)$ and brothel based than street or home based $(p<0.001)$ reported high degree of collective efficacy for addressing specific problems.

Results in table 3 indicate that FSWs reporting higher collective efficacy as compared with those with lower collective efficacy for achieving goals were more likely to negotiate condom use with occasional clients (60.3\% vs $19.7 \%$; adjusted OR (AOR) $=6.3,95 \%$ CI 4.8 to 8.4$)$ as well as regular partners $(62.8 \%$ vs $20.2 \% ; \mathrm{AOR}=6.4,95 \%$ CI 4.9 to 8.4 ). Similarly, FSWs who reported collection efficacy for addressing specific problems were more likely to negotiate condom use with occasional clients as well as regular partners. Additionally, FSWs who reported collective efficacy for addressing specific problems were more likely to report the ability to turn away clients when they were unwilling to use a condom as compared with those who did not report collective efficacy for addressing specific problems $(70.8 \%$ vs $58.4 \%$; $\mathrm{AOR}=1.8,95 \%$ CI 1.4 to 2.2 ). 
Table 2 Degree of collective efficacy for achieving certain goals and collective efficacy for addressing specific problems by background characteristics of female sex workers in Mumbai and Thane, India, 2010-2011

\begin{tabular}{|c|c|c|c|c|c|c|c|c|}
\hline \multirow[b]{2}{*}{ Background characteristics } & \multirow[b]{2}{*}{ Total sample } & \multicolumn{4}{|c|}{ Collective efficacy for achieving certain goals } & \multicolumn{3}{|c|}{$\begin{array}{l}\text { Collective efficacy for addressing } \\
\text { specific problems }\end{array}$} \\
\hline & & Low (\%) & Medium (\%) & High (\%) & p Value* & High (\%) & Low (\%) & p Value* \\
\hline Current age (years) & & & & & 0.392 & & & 0.198 \\
\hline $18-29$ & 1230 & 27.7 & 46.2 & 26.1 & & 73.9 & 26.1 & \\
\hline $30+$ & 876 & 30.1 & 45.8 & 24.1 & & 71.3 & 28.7 & \\
\hline Education & & & & & 0.265 & & & 0.005 \\
\hline No formal education & 1487 & 27.8 & 47.0 & 25.2 & & 74.6 & 25.4 & \\
\hline Formal education & 619 & 31.0 & 43.6 & 25.4 & & 68.7 & 31.3 & \\
\hline Marital status & & & & & $<0.001$ & & & 0.001 \\
\hline Never married & 384 & 31.5 & 44.3 & 24.2 & & 71.0 & 29.0 & \\
\hline Currently married & 808 & 34.7 & 38.5 & 26.9 & & 69.1 & 30.9 & \\
\hline Formerly married & 914 & 22.3 & 53.4 & 24.3 & & 76.8 & 23.2 & \\
\hline Duration of sex work (years) & & & & & 0.255 & & & 0.842 \\
\hline$\leq 2$ & 406 & 28.6 & 49.9 & 21.5 & & 73.8 & 26.2 & \\
\hline $3-5$ & 898 & 29.8 & 44.9 & 25.3 & & 72.3 & 27.7 & \\
\hline$\geq 6$ & 802 & 27.7 & 45.3 & 27.1 & & 72.9 & 27.1 & \\
\hline Typology† & & & & & $<0.001$ & & & $<0.001$ \\
\hline Brothel based & 753 & 27.8 & 51.2 & 21.0 & & 79.0 & 21.0 & \\
\hline Bar based & 703 & 21.2 & 50.1 & 28.7 & & 70.6 & 29.4 & \\
\hline Street/home based & 650 & 37.9 & 35.6 & 26.4 & & 68.0 & 32.0 & \\
\hline District & & & & & $<0.001$ & & & 0.547 \\
\hline Mumbai & 1064 & 34.1 & 38.3 & 27.7 & & 72.2 & 27.8 & \\
\hline Thane & 1042 & 23.3 & 53.9 & 22.8 & & 73.4 & 26.6 & \\
\hline $\begin{array}{l}\text { Collective efficacy for } \\
\text { achieving goals }\end{array}$ & & & & & & & & $<0.001$ \\
\hline Low & 605 & & & & & 58.5 & 41.5 & \\
\hline Medium & 969 & & & & & 72.7 & 27.3 & \\
\hline High & 532 & & & & & 89.3 & 10.7 & \\
\hline $\begin{array}{l}\text { Collective efficacy for addressing } \\
\text { specific problems }\end{array}$ & & & & & $<0.001$ & & & \\
\hline High & 1533 & 23.1 & 45.9 & 31.0 & & & & \\
\hline Low & 573 & 41.5 & 46.2 & 9.9 & & & & \\
\hline
\end{tabular}

*Differences in percentages between the categories were tested for statistical significance using the $\chi^{2}$ test.

†Typology refers to the usual place of solicitation for clients.

Results in table 4 indicate that FSWs who reported high degree of collective efficacy for achieving goals were more likely to be confident in: standing up to the police, madams/ brokers to help other FSWs $(5.8 \%$ vs $3.3 \%$; AOR $=2.7,95 \%$ CI 1.5 to 4.9), facing troublesome service providers
(73.5\% vs $38.8 \% ; \mathrm{AOR}=4.3,95 \%$ CI 3.3 to 5.6 ), supporting fellow FSWs in a crisis $(76.1 \%$ vs $49.6 \%$; AOR $=2.9,95 \%$ CI 2.2 to 3.7$)$ and had received help from other FSWs when a client or partner was violent $(73.9 \%$ vs $46.3 \%$; $\mathrm{AOR}=3.5,95 \%$ CI 2.7 to 4.5$)$.

Table 3 Self-efficacy for condom use by degree of collective efficacy for achieving certain goals and collective efficacy for addressing specific problems among female sex workers in Mumbai and Thane, India, 2010-2011

\begin{tabular}{|c|c|c|c|c|c|c|}
\hline Measures & Total $(\mathrm{N}=\mathbf{2 1 0 6})$ & \multicolumn{3}{|c|}{ Collective efficacy for achieving certain goals } & \multicolumn{2}{|c|}{$\begin{array}{l}\text { Collective efficacy for addressing } \\
\text { specific problems }\end{array}$} \\
\hline \multicolumn{7}{|c|}{$\begin{array}{l}\text { Self-efficacy in condom } \\
\text { use with clients* }\end{array}$} \\
\hline$\%$ & 45.6 & 19.7 & 53.7 & 60.3 & 38.1 & 48.3 \\
\hline AOR $(95 \% \mathrm{Cl})$ & - & Referent & 4.7 (3.7 to 6.1$)$ & $6.3(4.8$ to 8.4$)$ & Referent & $1.5(1.2$ to 1.8$)$ \\
\hline \multicolumn{7}{|c|}{$\begin{array}{l}\text { Self-efficacy in condom } \\
\text { use with regular partners } \dagger\end{array}$} \\
\hline AOR $(95 \% \mathrm{Cl})$ & - & Referent & 4.1 (3.2 to 5.2 ) & $6.4(4.9$ to 8.4$)$ & Referent & $1.4(1.1$ to 1.7$)$ \\
\hline \multicolumn{7}{|c|}{$\begin{array}{l}\text { Turned away clients when } \\
\text { they refused to use a condom }\end{array}$} \\
\hline$\%$ & 67.4 & 85.9 & 50.2 & 78.0 & 58.4 & 70.8 \\
\hline AOR $(95 \% \mathrm{Cl})$ & - & Referent & $0.2(0.1$ to 0.2$)$ & $0.6(0.5$ to 0.8$)$ & Referent & 1.8 (1.4 to 2.2$)$ \\
\hline
\end{tabular}

Variables controlled for in the logistic regression analyses are age, education, marital status, duration into sex work, typology and study district.

${ }^{*}$ Self-efficacy for condom use with clients was calculated from the following questions: How confident are you that you can use a condom with each client when (1) he gets angry with you, (2) he offers you more money for sex without a condom, (3) you have been using alcohol or drugs.

†Self-efficacy for condom use with regular partners was calculated from the following questions: How confident are you that you can use a condom with each regular partner when (1) he gets angry with you, (2) you think the risk of disease is low, (3) you have been using alcohol or drugs.

AOR, adjusted ORs; N, total sample size in that category. 
Table 4 Ability to handle crisis by degree of collective efficacy for achieving certain goals and for addressing specific problems among female sex workers in Mumbai and Thane, India, 2010-2011

\begin{tabular}{|c|c|c|c|c|c|c|}
\hline Ability to handle crisis & Total $(\mathrm{N}=2106)$ & \multicolumn{3}{|c|}{ Collective efficacy for achieving certain goals } & \multicolumn{2}{|c|}{$\begin{array}{l}\text { Collective efficacy for addressing } \\
\text { specific problems }\end{array}$} \\
\hline \multicolumn{7}{|c|}{$\begin{array}{l}\text { Stood up against the police and madams/ } \\
\text { brokers to help fellow FSWs in the past } \\
1 \text { year* }\end{array}$} \\
\hline$\%$ & 4.4 & 3.3 & 4.3 & 5.8 & 5.9 & 3.8 \\
\hline OR (95\% Cl) & - & Referent & $1.6(0.9$ to 2.8$)$ & $2.7(1.5$ to 4.9$)$ & Referent & $0.7(0.4$ to 1.1$)$ \\
\hline$\%$ & 65.9 & 38.8 & 78.6 & 73.5 & 53.7 & 70.4 \\
\hline OR (95\% Cl) & - & Referent & $6.4(5.0$ to 8.2$)$ & $4.3(3.3$ to 5.6$)$ & Referent & $2.3(1.9$ to 2.8$)$ \\
\hline \multicolumn{7}{|c|}{$\begin{array}{l}\text { Confident in supporting fellow FSWs } \\
\text { in crisis }\end{array}$} \\
\hline$\%$ & 71.6 & 49.6 & 83.0 & 76.1 & 62.8 & 75.0 \\
\hline OR (95\% Cl) & - & Referent & $4.4(3.4$ to 5.6$)$ & $2.9(2.2$ to 3.7$)$ & Referent & 1.9 (1.6 to 2.4$)$ \\
\hline
\end{tabular}

Variables controlled for in the logistic regression analyses are age, education, marital status, duration into sex work, typology and study district.

${ }^{*}$ Refers to the actual execution of behaviour.

AOR, adjusted ORs; FSW, female sex worker; N, total sample size in that category.

FSWs who reported a high degree of collective efficacy for achieving goals as compared with its counterpart were more likely to be confident in saying no to a client with whom they were unwilling to have sex (84.2\% vs $65.3 \%$; $\mathrm{AOR}=2.7,95 \% \mathrm{CI}$ 2.0 to 3.6 ), travelling alone (78.6\% vs $60.2 \%$; $\mathrm{AOR}=2.1,95 \% \mathrm{CI}$ 1.6 to 2.8$)$ and voicing their opinion in front of a large group (83.6\% vs 65.8\%; $\mathrm{AOR}=2.4$, 95\% CI 1.7 to 3.2 ) (table 5). Similarly, FSWs who reported collective efficacy for addressing specific problems as compared with its counterpart were more likely to have confidence in: saying no to a client with whom they were unwilling to have sex $(82.3 \%$ vs $70.2 \%$; $A O R=2.1$, $95 \%$ CI 1.6 to 2.6), going to a service provider and disclosing their identity as a FSW (81.6\% vs $65.4 \%$; AOR $=2.7,95 \%$ CI 2.1 to 3.4 ), travelling alone (74.9\% vs $70.3 \%$; AOR $=1.4,95 \%$ CI 1.1 to 1.8$)$, giving advice to others ( $80.0 \%$ vs $69.8 \%$; $A O R=1.9,95 \%$ CI 1.5 to 2.4 ) and voicing their opinion in front of a large group (81.4\% vs $72.4 \%$; $\mathrm{AOR}=1.8,95 \%$ CI 1.4 to 2.3 ).

The measure of overall individual empowerment is significantly associated with both the measures of collective efficacy. FSWs reporting high degree of collective efficacy for achieving goals were more likely to report greater empowerment $(66.7 \%$ vs $29.0 \%$; $\mathrm{AOR}=4.7,95 \%$ CI 3.6 to 6.2 ) than those who reported low collective efficacy (table 6). FSWs reporting collective efficacy for addressing specific problems were more likely to report greater empowerment than those reporting no/low such collective efficacy (56.0\% vs $47.0 \%$; $\mathrm{AOR}=1.5,95 \%$ CI 1.2 to 1.9 ).

Table 5 Factors affecting female sex workers' ability to negotiate for safe sex by degree of collective efficacy for achieving certain goals and for addressing specific problems) in Mumbai and Thane, India, 2010-2011

\begin{tabular}{|c|c|c|c|c|c|c|}
\hline \multirow[b]{2}{*}{ Self-confidence measures } & \multirow[b]{2}{*}{ Total $(N=2106)$} & \multicolumn{3}{|c|}{ Collective efficacy for achieving certain goals } & \multicolumn{2}{|c|}{$\begin{array}{l}\text { Collective efficacy for addressing } \\
\text { specific problems }\end{array}$} \\
\hline & & Low $(N=605)$ & Medium $(\mathrm{N}=969)$ & High $(\mathrm{N}=532)$ & Low $(N=572)$ & High $(\mathrm{N}=1534)$ \\
\hline \multicolumn{7}{|c|}{$\begin{array}{l}\text { Confident in saying no to a client with } \\
\text { whom she is unwilling to have sex }\end{array}$} \\
\hline$\%$ & 79.0 & 65.3 & 82.8 & 84.2 & 70.2 & 82.3 \\
\hline $\mathrm{OR}(95 \% \mathrm{Cl})$ & & Referent & $2.8(2.2$ to 3.7$)$ & $2.7(2.0$ to 3.6$)$ & Referent & $2.1(1.6$ to 2.6$)$ \\
\hline \multicolumn{7}{|c|}{$\begin{array}{l}\text { Confident in going to a service provider } \\
\text { and disclosing her identity as an FSW }\end{array}$} \\
\hline$\%$ & 77.2 & 70.4 & 80.8 & 78.6 & 65.4 & 81.6 \\
\hline OR (95\% CI) & & Referent & $1.6(1.3$ to 2.1$)$ & $1.3(0.9$ to 1.7$)$ & Referent & $2.7(2.1$ to 3.4$)$ \\
\hline \multicolumn{7}{|l|}{ Confident in travelling alone } \\
\hline$\%$ & 73.6 & 60.2 & 79.4 & 78.6 & 70.3 & 74.9 \\
\hline OR (95\% Cl) & & Referent & $2.3(1.8$ to 3.1$)$ & 2.1 (1.6 to 2.8 ) & Referent & 1.4 (1.1 to 1.8$)$ \\
\hline \multicolumn{7}{|c|}{$\begin{array}{l}\text { Confident in giving advice to neighbours } \\
\text { and other FSWs }\end{array}$} \\
\hline$\%$ & 77.2 & 64.5 & 82.8 & 81.8 & 69.8 & 80.0 \\
\hline OR (95\% Cl) & & Referent & $2.4(1.8$ to 3.0$)$ & 2.2 (1.7 to 2.9$)$ & Referent & 1.9 (1.5 to 2.4$)$ \\
\hline \multicolumn{7}{|c|}{$\begin{array}{l}\text { Confident in voicing an opinion in front } \\
\text { of a large group }\end{array}$} \\
\hline$\%$ & 78.9 & 65.8 & 84.5 & 83.6 & 72.4 & 81.4 \\
\hline OR (95\% Cl) & & Referent & 2.5 (1.9 to 3.3$)$ & 2.4 (1.7 to 3.2 ) & Referent & 1.8 (1.4 to 2.3$)$ \\
\hline
\end{tabular}

Variables controlled for in the logistic regression analyses are: age, education, marital status, duration into sex work, typology and study district.

AOR, adjusted ORs; FSW, female sex worker; N, total sample size in that category. 
Table 6 Overall empowerment by degree of collective efficacy for achieving certain goals and for addressing specific problems among female sex workers in Mumbai and Thane, India, 2010-11

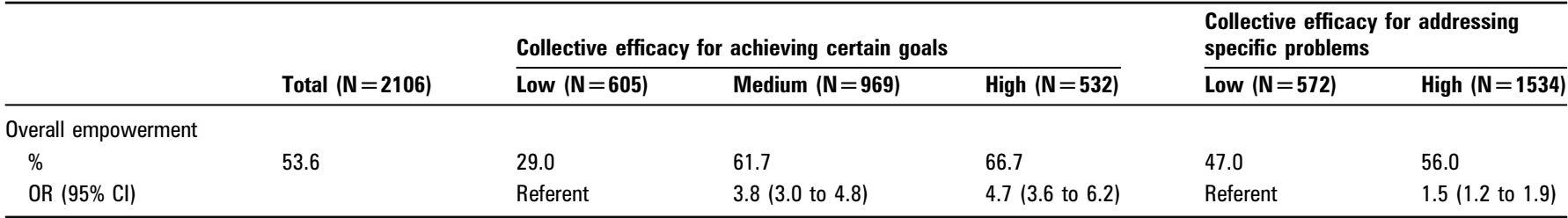

Variables controlled for in the logistic regression analyses are age, education, marital status, duration into sex work, typology and study district. The summary measure of empowerment was constructed by averaging the responses to the 12 questions used for the dimensions of self- efficacy and self-confidence: her ability to use condoms, negotiate condom use, handle crises, utilise resources, identify herself as a sex worker in a group, and confidence in giving her opinion in front of a large group. The score varied from 1 to 2 , which had reliability (Cronbach's alpha) of 0.78. The index score was further divided into two equal categories of overall empowerment: low (1-1.4999) and high (1.50-2.0).

AOR, adjusted ORs; $N$, total sample size in that category.

\section{DISCUSSION}

This cross-sectional investigation of association between collectivisation and empowerment-related indicators for FSWs indicates that those reporting higher collective efficacy are also more likely to report higher self-efficacy in condom use with clients and regular partners and their ability to turn away clients when they refuse to use condoms. This demonstrated relationship between collectivisation, collective efficacy and the indicators of self-efficacy, and confidence gains importance given that FSWs are highly mobile and face multiple vulnerabilities associated with duration of sex work and educational background in cities like Mumbai and Thane. ${ }^{19}$ Importantly, findings from the current study underline the importance of collective efficacy as strategy to promote HIV prevention interventions, especially in challenging settings as evidenced in the Aastha project and elsewhere. $^{22} 23$ These results parallel the previous research findings in Karnataka where it was shown that a higher degree of collectivisation was associated with increased knowledge and condom use by FSWs, ${ }^{5}$ and in Andhra Pradesh, where it was evident that higher degree of community mobilisation increased condom use and reduce violence. ${ }^{122}$

Findings of this study also provide empirical evidence of the relationship between FSWs collectivisation and their confidence in standing up to the police and troublesome stakeholders if there were any issues that affected the FSW community. These results indicate much more benefits in an HIV prevention initiative than mere promotion of safe sex behaviours. Twothirds of FSWs reported medium or high collective efficacy for achieving goals, which can be attributed to the support systems developed across different levels of the Aastha Parivaar and the continuous support provided by Aastha CBOs to AGs at the local level. The systems developed in the project also included building FSWs' knowledge and capacity to provide support promptly to each other. FSW collectivisation, as envisaged under the Aastha community mobilisation strategy, resulted in their enhanced ability to manage crisis situations independently and stand up to power structures, including brothel keepers, the police and brokers. ${ }^{17}$ These findings support previous research results which report that interventions using peer education for FSWs could improve their daily lives. ${ }^{26} 27$ The findings that FSWs are willing to speak in public, are not afraid of travelling alone and are able to give advice to fellow FSWs show the correlation between collectivisation and higher confidence levels.

The study results on the association between collective efficacy for addressing specific problems and FSWs increased selfconfidence in facing troublesome stakeholders or service providers independently and also helping fellow sex workers in crises situations reinforce the need for building relationship bonds between FSWs and that they should be initiated in the early stages of the programme. While it is difficult to determine whether collective efficacy per se actually helped in building FSWs' self-confidence on many issues; however, the data show that FSWs' confidence in expression of thoughts and feelings/ speaking publicly is perhaps a consequence of collectivisation. Programme experience suggests that in the early stages of the intervention, FSWs rarely communicated with each other, let alone coming together for any purpose or event. Most FSWs considered each other as rivals, which is believed to have compounded their sense of isolation created by being from different cultural backgrounds. During this period, the project emphasised the need to collectivise FSWs into AGs, which would provide a forum to discuss and solve individual as well as group issues. Following these efforts, FSWs realised they had common issues and began to experience group bonding. The knowledge of having a support system which they could access in case of a crisis situation greatly contributed to building their self-confidence and self-efficacy, and individual FSWs began to insist on condom use with clients or resist violence with the belief that the larger group would intervene if needed. These descriptions from the programme and our study results reinforce the need for developing collective groups and communicating on the benefits of such collectivisation.

Although the empirical evidence on the linkages between collectivisation and self-efficacy and confidence has several important programmatic implications, the results of this study must be interpreted cautiously in light of several limitations. First, responses to reported collective efficacy and self-confidence outcomes are based on self-reports and may therefore be vulnerable to significant social desirability and reporting biases. The use of experienced research staff linked with training and local support was designed to increase respondents' comfort and reduce social desirability; moreover, the use of short time-frames for questions was used to indicate their current status on all the indicators used. Second, the analyses are cross-sectional; thus, causality cannot be assumed in linkages between degree of reported collective efficacy and empowerment of FSWs. While this provides useful information in terms of the association between the indicators of self-efficacy and self-confidence with collective efficacy, further longitudinal research is needed to understand the causality between the two sets of measures used in the study. Due to this limitation, the findings of this study refer to the association between the degree of perceived collectivisation and empowerment of FSWs and not to how collectivisation per se led to increased empowerment of FSWs. Third, this study was conducted in Mumbai and Thane districts of Maharashtra, which are both highly industrial, economic hubs of commerce as well as migration destinations that have also seen a significant churn rate of FSWs. The sex work setting in these two industrial districts is highly heterogeneous and may be different from sex work settings in other cities, states or 


\section{What is already known on this subject}

- Community collectives can empower vulnerable populations to negotiate safe sex practices.

- Empowerment strategies have been successful with homogenous groups of sex workers.

\section{What this study adds}

- Community collective efficacy is strongly associated with FSWs' increased confidence in times of crisis and increased self-efficacy for condom use.

- Collective efficacy for achieving goals, addressing specific problems and the associated self-efficacy within HIV prevention intervention is a possibility even in diverse settings through a context-specific grass-root-level programme planning and implementation.

- Community collectivisation through smaller groups (two to three self-help groups for every 50 FSWs), expanding to become community-based organisations and then a federation is a bottom-to-top approach in the scaling-up of HIV prevention interventions.

provinces and countries; hence, the results may not be generalisable and solely refers to the study districts. Finally, the independent associations measured between degree of collectivisation and the empowerment of FSWs could also question the adequacy of the items included in each of the measures. While future studies could address some of the issues related to measurement of collectivisation, this large-scale study highlights the relationship between collective efficacy and selfefficacy and self-confidence among FSWs who belong to different states of the country.

In summary, the results of this study suggest that FSWs who perceived high degree of collectivisation were more likely to also report self-efficacy for condom use and self-confidence in handling crisis situations. The Aastha intervention conducted with a heterogeneous group of FSWs belonging to different regions, religions and social sectors adapted the strategy of creating smaller groups at the local level that helped mobilise the community and formation of community-based organisation at the macro level. The model may be relevant in other national settings but focused research with a longitudinal study design is needed to document the pathways or mechanisms for the effect of collectivisation on individuals' empowerment.

Acknowledgements Since 2004, FHI 360 (brand name for Family Health International, FHI) has been implementing the Aastha project with the goal to reduce the incidence of STIs and HIV among FSWs and their regular partners in the Mumbai and Thane districts of Maharashtra state in India. The authors wish to acknowledge the Aastha project team from FHI 360, Family Planning Association of India (FPAl), Society for Service to Voluntary Agencies (SOSVA) and the Implementing Partners and all the sex workers who participated in the study. We acknowledge the contributions of the unnamed reviewers in strengthening the paper.

Contributors SSG led the study conception, analyses and manuscript writing. $A B$ assisted with the study conception and manuscript writing. VR and NS designed the analytical approach, analyses and assisted with manuscript writing. GN assisted with interpretation of study findings and with lessons learnt from the project.
Funding The Aastha project is funded by the Bill \& Melinda Gates Foundation through Avahan, its India AIDS Initiative. However, the views expressed herein are those of the authors and do not reflect the official policy or position of the Bill \& Melinda Gates Foundation and Avahan.

\section{Competing interests None.}

Ethics approval Ethics approval was provided by FHI 360's Protection of Human Subjects Committee (PHSC) and a local Community Advisory Board.

Provenance and peer review Commissioned; externally peer reviewed.

\section{REFERENCES}

1. Blankenship KM, West BS, Kershaw TS, et al. Power, community mobilization, and condom use practices among female sex workers in Andhra Pradesh, India. AIDS 2008:22:S109-16.

2. Parker RG. Empowerment, community mobilization and social change in the face of HIV/AIDS. AIDS 1996:10:S27-31.

3. Evans C, Jana S, Lambert H. What makes a structural intervention? Reducing vulnerability to HIV in community settings, with particular reference to sex work. Glob Public Health 2010;5:449-61.

4. Evans C, Lambert H. Implementing community interventions for HIV prevention: insights from project ethnography. Soc Sci Med 2008;66:467-78.

5. Halli SS, Ramesh BM, O'Neil J, et al. The role of collectives in STI and HIV/AIDS prevention among female sex workers in Karnataka, India. AIDS Care 2006;18:739-49.

6. Swendeman D, Basu I, Das S, et al. Empowering sex workers in India to reduce vulnerability to HIV and sexually transmitted diseases. Soc Sci Med 2009:69:1157-66.

7. Cornish F. Challenging the stigma of sex work in India: material context and symbolic change. J Community Appl Soc Psychol 2006;16:462-71.

8. Jana S, Bandyopadhyay N, Mukherjee S, et al. STD/HIV intervention with sex workers in West Bengal, India. AIDS 1998;12:S101-8.

9. Jana S, Singh S. Beyond medical model of STD intervention-lessons from Sonagachi. Indian J Public Health 1995:39:125-31.

10. Nag M. Sex workers in Sonagachi-pioneers of a revolution. Econ Political Weekly 2005:40:5151-6

11. Nath MB. Women's health and HIV: experience from a sex workers' project in Calcutta. Gend Dev 2000;8:100-8.

12. Cornish F, Ghosh R. The necessary contradictions of 'community-led' health promotion: a case study of HIV prevention in an Indian red light district. Soc Sci Med 2007;64:496-507.

13. Bandopadhyay N, Ray K, Baneriee A. Operationalizing an Effective Community Development Intervention for Reducing HIV Vulnerability in Female Sex Work: Lessons learned from the Sonagachi project in Kolkata, India. International Conference on AIDS. Barcelona, Spain: International AIDS Society (IAS), 2002.

14. Jana S, Basu I, Rotheram-Borus MJ, et al. The Sonagachi Project: a sustainable community intervention program. AIDS Educ Prev 2004;16:405-14.

15. Busza J. Participatory research in constrained settings. Action Res 2004;2:191-208

16. India Health Action Trust (IHAT). HIV/AIDS Situation and Response in Maharashtra: Epidemiological Appraisal Using Data Triangulation. Bangalore, India: IHAT, NIMS, 2011:23-4, 72 .

17. Wheeler T, Kiran U, Jayaram M, et al. Live and learn: reflections on participation and community mobilization through the implementation of the Avahan HIV/AIDS Initiative in India. J Epidemiol Community Health 2012;66: ii16-ii25.

18. Schensul SL, Saggurti N, Singh R, et al. Multilevel perspectives on community intervention: an example from an Indo-US HIV prevention project in Mumbai, India. Am J Community Psychol 2009:43:277-91.

19. Saggurti N, Jain A, Sebastian M, et al. Indicators of mobility, socio-economic vulnerabilities and HIV risk behaviours among mobile female sex workers in India. AIDS Behav 2012;16:952-9.

20. National AIDS Control Organization (NACO). HIV Sentinel Surveillance and HIV Estimation In India 2007 A Technical Brief. New Delhi: NACO, Ministry of Health and Family Welfare, Government of India, 2008.

21. Indian Council of Medical Research (ICMR), Family Health International (FHI). National summary Report: Integrated Behavioral and Biological Assessment, 2009. Pune, India: National AIDS Research Institute, 2010.

22. Punyam S, Pullikalu RS, Mishra RM, et al. Community advocacy groups as a means to address the social environment of female sex workers: a case study in Andhra Pradesh, India. J Epidemiol Community Health 2012;66:ii87-ii94.

23. Chakravarthy JBR, Joseph SV, Pertti P, et al. Community mobilisation programme for female sex workers in coastal Andhra Pradesh, India: processes and their effects. J Epidemiol Community Health 2012;66:ii78-ii86,

24. Swisher RR. Neighborhoods. In: Salkind NJ, ed. Encyclopedia of Human Development. Thousand Oaks, CA: Sage Publications, 2005.

25. Cox DW. Self-efficacy. In: Salkind NJ, ed. Encyclopedia of Human Development Thousand Oaks, CA: Sage Publications, 2005

26. Argento E, Reza-Paul S, Lorway R, et al. Confronting structural violence in sex work: lessons from a community-led HIV prevention project in Mysore, India. AIDS Care 2011:23:69-74.

27. Rekart ML. Sex-work harm reduction. Lancet 2005;366:2123-34. 